\title{
La formación de los profesores de Filosofía u la paradoja del "giro filosófico"
}

\section{A formação dos professores de Filosofia e o paradoxo do "giro filosófico"}

\section{Formation of teachers of Philosophy and the paradox of "turn philosophical"}

\author{
Gustavo Ruggiero ${ }^{1}$
}

\begin{abstract}
RESUMEN
En el presente artículo se recupera la lectura de un texto del profesor Guillermo Obiols con el doble propósito de mantener viva la memoria de una búsqueda filosófica colectiva e intentar a su vez un balance de nuestras prácticas de enseñanza de la Filosofía. Se propone también pensar la formación de los futuros profesores de Filosofía desde una perspectiva filosófica que asuma la centralidad del proyecto de autonomía como horizonte de un trabajo de reconstrucción de la propia subjetividad.
\end{abstract}

Palabras-clave: giro filosófico; autonomía; elucidación.

\section{RESUMO}

Este artigo recupera a leitura de um texto do professor Guillermo Obiols com o duplo propósito de manter viva a memória de uma busca filosófica coletiva e promover um balanço de nossas práticas de ensino de Filosofia. Também tem o propósito de pensar a formação dos futuros professores de Filosofia desde uma perspectiva filosófica que assuma a centralidade do projeto de autonomia como horizonte de um trabalho de reconstrução de sua própria subjetividade.

Palavras-chave: giro filosófico; autonomia; elucidação.

${ }^{1}$ Doctor en Filosofía. Profesor de la Universidad Nacional de General Sarmiento (UNGS), Argentina. 


\begin{abstract}
This article recovers the reading of a text by professor Guillermo Obiols with the dual purpose of keeping the memory of a collective philosophical search alive and, in turn, trying to balance our Philosophy teaching practices. There is also a proposal to consider the qualification of future Philosophy teachers from a philosophical perspective that assumes the core of the autonomy project as the basis of the reconstruction work on subjectivity itself.
\end{abstract}

Keywords: philosophical change; autonomy; clarification.

\title{
Introducción
}

Hace veinte años, Guillermo Obiols, referente indiscutido y precursor de lo que hoy podemos señalar con precisión - dentro y fuera de la academia - como el campo de la didáctica de la Filosofía en Argentina, escribió un texto con magistral sencillez donde anunciaba que el problema principal para la enseñanza de la Filosofía no era un problema de método. En ese texto, escrito a partir de las dificultades que la formación de profesores de Filosofía debe enfrentar, Obiols indicaba cuatro situaciones - que las destacamos a su vez como punto de partida de esta reflexión - sobre las que era necesario trabajar:

1 - El poco aprecio de los estudiantes de las carreras de Filosofía por la enseñanza en general;

2 - La necesidad de definir la Filosofía en nombre de la cual se va a enseñar;

3 - La problematización del concepto mismo de enseñanza;

4 - La relación entre enseñanza y Filosofía.

Proponemos retomar estas cuestiones, luego de transcurrido un tiempo considerable y de contar a su vez con muchísimas reflexiones colectivas, producciones teóricas diversas y experiencias pedagógicas y filosóficas de lo más variadas que buscaron en general renovar las aulas de Filosofía de la escuela secundaria, para evaluar dónde estamos hoy, cuánto se pudo progresar respecto de los desafíos propuestos por la didáctica de la Filosofía y pensar finalmente los límites y condiciones que encontramos en la tarea de enseñar Filosofía y en la de formar a quienes van a enseñarla. Intentaremos posteriormente poner este análisis en una perspectiva filosófica y política que excede el marco de la didáctica especial para asumir en última instancia los problemas y desafíos de la educación institucionalizada. 


\section{La didáctica de la Filosofía y la paradoja del giro filosófico}

Aunque parezca ajeno a nuestro empeño en pensar sobre la transmisión institucionalizada de la Filosofía, en Argentina, la aparición de publicaciones y la organización de jornadas y encuentros referidos a los problemas teóricos y metodológicos de la enseñanza de la Filosofía, tienen como destacado correlato el reinicio de los gobiernos constitucionales y el retorno de la democracia. Curiosamente, al igual que en la España franquista, durante la horrorosa dictadura militar (1976-1983), la materia Filosofía continuó dictándose en la escuela secundaria argentina. Sería asunto de una reflexión aparte - y por cierto muy interesante - comprender las razones por las cuáles dos gobiernos, en extremo autoritarios, no suprimieron nuestra asignatura como si lo hicieron con otras vinculadas a las humanidades o las ciencias sociales.

Tenemos entonces que en Argentina el inicio de lo que puede considerarse como el giro didáctico de la enseñanza de la Filosofía, puede datarse a partir de los años 80 con los trabajos de algunos profesores, entre los cuales destacan los de Guillermo Obiols, y es coincidente con la consolidación de la didáctica de la Filosofía como un campo disciplinar reconocible (OBIOLS; RABOSSI, 2000).

La lectura de aquellos textos de Obiols nos ha enseñado varias cosas. Particularmente a quienes nos dedicamos a "formar" profesores de Filosofía, estos trabajos ofrecen algunos esquemas prácticos que nos permiten enfocar la enseñanza de la enseñanza de la Filosofía de un modo bastante claro. Uno de esos esquemas, al que aquí nos remitimos, es el que a comienzos de los 90 Obiols tituló, en un artículo titulado "Una misión casi imposible: enseñar a enseñar Filosofía", que compone un libro pionero escrito junto a Marta Frassineti.

El segundo de esos esquemas prácticos, una suerte de estado del arte, es el que escribió junto a Laura Agratti: "Concepciones de la Filosofía y enseñanza de la Filosofía en la escuela secundaria: un análisis a través de los programas y los libros de texto".

Ambos trabajos nos han sido muy útiles en estos años para enfocar la formación de los profesores de Filosofía, tanto en una perspectiva filosófica propiamente dicha, como en una perspectiva histórica y pedagógica.

Del primero de los textos, nos será provechoso volver a pensar las cuatro dificultades que Obiols encontraba en esta tarea. Del segundo, tomamos la progresión histórica de las influencias que las distintas concepciones filosóficas ejercieron sobre la enseñanza de la Filosofía en la escuela secundaria argentina. Dicha influencia es indagada por los autores, especialmente en los libros de texto que desde fines del siglo XIX y durante todo el siglo XX se editaron a propósito 
de tal cometido. Mencionamos este texto porque nos interesa dejar planteada, siguiendo la idea que los autores proponen sobre la sucesiva progresión de los paradigmas que orientaron la producción de manuales para la enseñanza de la Filosofía en la secundaria, la pregunta sobre cuál es "el paradigma actual" que orienta las prácticas de enseñanza de la Filosofía. Si podemos tomar por cierto que a partir de los años 1980, el propio Obiols junto a otros profesores, da lugar a un giro didáctico en el campo de la enseñanza de la Filosofía, lo que aquí propongo es saber si podríamos hablar en la actualidad de un giro filosófico con relación a la investigación teórica y metodológica en torno a la enseñanza de la Filosofía. Los estudios más recientes sobre la enseñanza de la Filosofía han ido dando, progresivamente, un perfil singular a su horizonte problemático, ampliando la concepción tradicional y el alcance de la "didáctica de la filosofía", vinculándola más con desarrollos propios del campo filosófico (GALLO, 2003; LANGON, 2003; CERLETTI, 2008).

Es preciso señalar, no obstante, dos cuestiones. En primer lugar, el giro filosófico, si tal denominación fuera pertinente, puede deberse al propio movimiento institucional iniciado por Obiols pero no sólo por él. ${ }^{2} \mathrm{Y}$ en segundo término, este mismo giro, paradójicamente, debería ser relativizado si tomamos en consideración las prácticas efectivas de la enseñanza de la Filosofía.

La producción editorial de un conjunto creciente de artículos y libros que dan cuenta de nuevos modos de orientar la enseñanza de la Filosofía, contrasta efectivamente cuando consideramos lo que sucede hoy mismo en las aulas de la escuela secundaria donde se dicta la materia Filosofía. Tomamos como referencia inmediata para comenzar a pensar esta paradoja, lo que ha señalado muy bien P. Vicari (2009) en un artículo que analiza la enseñanza de la Filosofía y el curriculum provincial de la jurisdicción Buenos Aires y un estudio que realizamos desde un equipo de investigación en la Universidad Nacional de General Sarmiento.

Vicari indica, entre otros datos realmente valiosos, que al momento de crearse la materia Filosofía y Formación Ética y Ciudadana con la sanción de la Ley Federal de Educación (1993/1995), ${ }^{3}$ y al margen de todo lo que podemos

\footnotetext{
${ }^{2}$ Valen mencionar como muestra de los aportes de nuestra región, a riesgo de olvidar algunos nombres importantes, los trabajos de Olga Grau en Chile; Walter Kohan, Sergio Sardi, Silvio Gallo, Elisete Tomazetti, Geraldo Balduino en Brasil; Mauricio Langon, Marisa Berttolini, Isabel Gonzalez, Janett Tourn en Uruguay; Marta Frassineti, Alejandro Cerletti, Ana Couló, Laura Agratti, Verónica Bethencourt en Argentina, sólo por citar aportes destacados. Sabemos a su vez que encontraremos aportes de igual densidad en países como Francia, España o Italia, si fuera necesario demostrar que iguales preocupaciones han ocupado, en otras regiones, a los profesores de Filosofía.

${ }^{3}$ Posteriormente fue sancionada la nueva Ley Nacional de Educación, del año 2006, pero no se registran modificaciones sustanciales del escenario para la enseñanza de la Filosofía.
} 
discutir sólo sobre este hecho, se generó algo parecido a un "vacío" filosófico-pedagógico, desde el momento en que profesores sin título específico en Filosofía - esto es, abogados, profesores de historia, ciencias jurídicas o licenciados en ciencias de la educación - no siempre estuvieron dispuestos a problematizar filosóficamente (ni lo están aún en muchos casos) sus propuestas, como tampoco estuvieron espontáneamente dispuestos a problematizar filosóficamente las cuestiones sociopolíticas, aquellos profesores que sí portaban título específico en Filosofía. Para estos últimos, el precio de abandonar el "canon" parecía ser lo suficientemente elevado como para abordar nuevas prácticas.

El análisis de nuestro colega Vicari, al que por efecto de mi propia tarea docente en la escuela secundaria considero muy verosímil, puede completarse con los resultados de una investigación acreditada en la Universidad Nacional de General Sarmiento, llevada adelante durante los años 2005-2008 y dirigida Alejandro Cerletti. ${ }^{4}$ Dicha investigación tuvo como objetivo principal relevar la situación de la enseñanza de la Filosofía en el conurbano bonaerense. ${ }^{5}$ Entre un conjunto de datos que arrojó esta indagación, se pudo observar que la enseñanza de la Filosofía es una práctica sobre la que hay un insuficiente nivel de reflexión. Además de la ausencia de espacios específicos para que ello ocurra, la base empírica que nos proveyó la encuesta realizada a más de un centenar de profesores, indicó que tanto los objetivos que trazaban para sus clases, como los propósitos, las lecturas escogidas, las actividades que ofrecían a sus alumnos y los modos de evaluación, se ajustaban a prácticas mas bien "tradicionales", ajustadas con frecuencia a los lineamientos oficiales. El relato de los docentes - mayoritariamente - señalaba que las dificultades que encuentran para el desarrollo de sus clases recaen casi exclusivamente sobre los alumnos y sus más variados "déficits", antes que en las propias condiciones materiales de su tarea o, por caso, en la propia formación.

La práctica docente en Filosofía es, por lo general, una práctica bastante acrítica, vale decir, no pensada filosóficamente. Los profesores suelen justificar su tarea, oscilando entre un voluntarismo disciplinar universitario - cualquiera que sepa Filosofía puede enseñarla - y un dogmatismo que sostiene que enseñar Filosofía es transmitir un cuerpo de saberes ya establecidos y legitimados históricamente. En el otro extremo, encontramos a quienes piensan que el pro-

${ }^{4}$ Cf. Proyecto de Investigación: “Condiciones y posibilidades de la enseñanza de la Filosofía (un análisis filosófico, didáctico y político). El caso especial de la enseñanza de la Filosofía en el conurbano bonaerense". Instituto del Desarrollo Humano. Universidad Nacional de General Sarmiento.

${ }^{5}$ El conurbano bonaerense es una región de la provincia de Buenos Aires, densamente poblada, colindante con la Ciudad Autónoma de Buenos Aires, Capital Federal de la República Argentina. 
blema de la enseñanza de la Filosofía se resuelve sólo con algunas estrategias didácticas que harían enseñable prácticamente cualquier tema (CERLETTI; RUGGIERO, 2010).

Lo que queremos dejar planteado como problema - que puede abordarse tanto desde la didáctica de la Filosofía, como desde la Filosofía de la educación o desde la Filosofía misma - es la paradoja que proponemos a su vez como hipótesis: que existe una distancia entre la riqueza y diversidad de las propuestas teóricas y metodológicas del campo disciplinar de la didáctica de la Filosofía, que contrasta con la precariedad de las prácticas que un observador crítico registraría hoy en el aula de la escuela secundaria. Nos resta hacer el intento por comprender las razones de esta distancia y proponer el modo de acortarla o superarla.

\section{Hace veinte años que tenemos veinte años}

Tanto los profesores y profesoras de Filosofía actualmente en ejercicio, como los aspirantes a serlo, cuentan hoy, tras dos décadas de ininterrumpida producción teórica y metodológica, con una importantísima cantidad de libros, textos, encuentros, jornadas y talleres - e incluso sitios web con la más diversas perspectivas - que le permitirían abordar la reflexión sobre sus prácticas y diseñar y llevar a cabo propuestas más o menos novedosas para el aula. A pesar de ello, hemos dicho, la enseñanza de la Filosofía, salvo escasas excepciones, suele presentarse bajo propuestas no asumidas filosóficamente.

Repasemos una lista breve de aquellas preocupaciones que registramos año tras año entre los estudiantes de Filosofía que quieren convertirse en profesores, para ver si, entre estas representaciones sobre encuentro entre la Filosofía y su enseñanza y aprendizaje, hallamos algunas claves que nos permitan vislumbrar sobre qué condiciones es posible construir una didáctica filosófica. Una didáctica que para ser considerada "filosófica" suponemos a priori como autónoma. Vale decir, gestada desde la propia definición de Filosofía y de lo que significa enseñarla, asumiendo que será una actividad condicionada pero no determinada absolutamente por el campo de lo social-histórico. ${ }^{6}$

¿Qué cosas le inquietan a un futuro profesor de Filosofía?

- Adquirir habilidades para dictar una clase exitosamente.

\footnotetext{
${ }^{6}$ Pensamos aquí la autonomía desde las ideas desarrolladas por C. Castoriadis.
} 
- Compartir experiencias del aula como docente.

- Reencontrarse con lo aprendido durante la carrera para enseñarlo de la mejor manera posible.

- Aprender a secuenciar contenidos.

- Aprender a organizar los tiempos.

- Tener buen "manejo" de grupo.

- Capturar la atención de los alumnos

- No poder responder preguntas de los alumnos.

- No poder preparar una clase motivadora.

- Conocer la institución escolar y el sistema educativo.

- Saber planificar.

- Tener un buen método de enseñanza.

- Cómo hacer para transmitir la inquietud por el tema que se está enseñando.

- Desnaturalizar los saberes de los alumnos.

- Temor a descubrir que la enseñanza no sea lo propio.

Estas son algunas de las respuestas que aparecen con altísima frecuencia frente a la pregunta señalada. Entre ellas, algunas tienen un carácter más instrumental y otras se acercan a preocupaciones pedagógicas, y en algunas otras se insinúa una preocupación filosófica. Si enseñar Filosofía supone algunas dificultades que se derivan de la toma de decisiones fundamentales por parte del profesor/a, enseñar a enseñar Filosofía multiplica esas dificultades. Vistas las cosas desde el lado del profesor que forma profesores de Filosofía, nos encontramos con las siguientes preocupaciones al observar el trabajo de quienes están a un paso de ser profesores de Filosofía en la escuela secundaria:

- Distancia entre el discurso y la práctica. Clases que plantean como objetivo desarrollar un pensamiento crítico, pero muy centradas en la exposición del docente.

- Escasa autonomía respecto del diseño curricular y su posible reformulación.

- Las actividades se centran en la transmisión de conceptos.

- Las planificaciones no evidencian con claridad que la enseñanza de la Filosofía pueda ser un problema filosófico.

- Los enfoques de la enseñanza no están claros. En general no suelen ser problemáticos. Las actividades no alientan la construcción de problemas y se presentan los problemas ya tratados por los filósofos de modo ajeno a los propios intereses.

Hay un sentido común contra el que toda Filosofía, desde Sócrates en adelante, pretende luchar. Pero deberíamos advertir también que hay un sen- 
tido común filosófico contra el que todo estudiante de Filosofía (y también los profesores por supuesto) debe estar alerta.

Es probable que el título que Obiols escogió para su artículo no haya sido al pasar. Él decía allí que enseñar a enseñar Filosofía es una tarea casi imposible. Subrayamos este casi porque es posible que allí se encuentre la clave para que el diagnóstico sobre estas dificultades, que constituyen algo así como la ontología de la formación de los profesores/as de Filosofía, de lugar al devenir de otras subjetividades.

Repasemos una vez más las dificultades que señalaba Obiols. En primer término decía que los estudiantes de la carrera de Filosofía suelen tener en poca estima la enseñanza. La inclinación a pensar que hacer Filosofía se vincula a la investigación o a la lectura solitaria, sumada a la exigencia erudita de la cita de autoridad y a la acreditación académica de los papers, aleja a los estudiantes del gusto por la situación de enseñanza. Es probable que el propio discurso académico se constituya alrededor de la idea de que la docencia es un mal menor o un tiempo a pagar a cambio de la cercanía a la Filosofía. La investigación filosófica suele asociarse con la originalidad y la enseñanza con la repetición.

La segunda dificultad apunta a que cualquier enseñanza de la Filosofía depende de la concepción que de ella se tenga. Vale decir que el modo en que nos aproximemos a tal o cual problema filosófico lleva tácitamente una definición de la Filosofía, seamos o no concientes de ello. Cabe destacar que en torno a esta dificultad Cerletti (2008) ha construido una importante e insoslayable reflexión. El hecho de aprender a ser profesores mucho antes de ingresar al profesorado, condiciona en gran medida nuestro modo de ser docentes. Si no se trabaja previamente sobre la propia definición que hagamos de la Filosofía y el filosofar es muy probable que repitamos acríticamente las actitudes de aquellas maestras, profesoras y profesores, con quienes tuvimos la fortuna o la desdicha de encontrarnos en la vida. De hecho, el punto de partida que propone Cerletti para la formación de los profesores de Filosofía es justamente la creación de un espacio de acompañamiento y estímulo a los futuros profesores invitándolos a transitar la búsqueda de la respuesta personal a la pregunta sobre qué se entiende por Filosofía y qué por enseñarla (Cerletti, 2008).

En cuanto a la tercera y cuarta dificultades, estas refieren a los problemas que se derivan del concepto de enseñanza, por un lado - lo cual nos obliga como filósofos al diálogo con otras disciplinas como la pedagogía y la psicología - y a la intersección entre los conceptos de enseñanza y Filosofía, por otro: ¿qué quiere decir enseñar?, ¿qué quiere decir enseñar Filosofía?, ¿es la Filosofía enseñable?

Para cada una de estas dificultades, Obiols ensayó algunos recorridos en dirección a su superación. Desde el impulso a la creatividad que intentase des- 
plazar la simple repetición e hiciera más atractiva y estimulante la tarea docente; la adopción de un concepto amplio de Filosofía adoptado por consenso y que permitiese incluir las diferentes formas de entenderla; la definición también general y un tanto arbitraria de enseñanza, comprendida como la actividad que incluye la planificación, conducción y evaluación de actividades tendientes a producir un aprendizaje en los alumnos; o el examen de las distintas prácticas de enseñanza de la Filosofía antes que la discusión vacía sobre las condiciones de posibilidad de un enseñanza filosófica, todas respuestas, decíamos, que daban cuenta de un esfuerzo teórico-práctico que a juzgar por lo sucedido desde su escritura, hoy nos tiene aquí continuando la tarea.

Si hemos recuperado el texto de Obiols ha sido con el doble propósito de mantener viva la memoria de una búsqueda filosófica, que a esta altura podemos decir que se trata de un recorrido colectivo, pero también para hacer un balance de nuestras prácticas. Sospechamos que estas dificultades siguen vigentes. Por supuesto que hay un grado de universalidad logrado por Obiols en las preguntas que subyacen a cada dificultad, que hace posible su vigencia. Sin embargo, el objeto de esta reflexión apunta al examen de lo que ocurre cotidianamente en las aulas de la escuela secundaria. A juzgar por la ausencia de foros provinciales o nacionales que reúnan a profesores y profesoras de Filosofía, como sí ocurre en otros países y debido también a la ausencia de los mismos profesores en las jornadas académicas destinadas a la enseñanza de la Filosofía, sumado esto a la inestable permanencia de la materia en el plan de estudios y a la declinación de nuestra capacidad asociativa, la práctica de la enseñanza de la Filosofía no está en su mejor momento.

La abundancia de producción teórica sobre la enseñanza de la Filosofía contrasta, como dijimos, con la escasez de estudios sobre lo que ocurre en el aula. Hemos trabajado poco sobre estas dificultades desde el interior de la escuela. Quizás no tengamos espacio aquí para poner en contexto lo dicho y evaluar la relación que tienen las prácticas áulicas con la política educativa nacional acontecida en las últimas dos décadas. No es poco lo ocurrido en estas dos últimas décadas. Por otra parte, es muy probable, a su vez, que el abandono de las viejas prácticas enciclopédicas que marcaron los inicios de los sistemas educativos nacionales no haya devenido en una superación crítica de las mismas. Podría ser cierto, incluso, que nos encontremos con aulas donde se practique una enseñanza de la Filosofía tradicional pero devaluada en su aspecto disciplinar. O podría ser también cierto que, en nombre de la Filosofía, se entienda que al analizar una noticia de actualidad formulando algunas preguntas, estuviésemos filosofando. Sería de suma importancia para el futuro de la enseñanza de la Filosofía en la escuela secundaria encarar una investigación que de cuenta más precisa, de manera cualitativa y cuantitativa, de lo que ocurre al interior de las 
aulas. Es necesario comprender los "esquemas prácticos" de los docentes, las teorías implícitas en sus propuestas, sus valores y creencias pedagógicas, puesto que todos ellos forman, en su conjunto, un a priori en la tarea docente.

La percepción de las prácticas que aquí hemos señalado con cierta aspereza provienen de varios años de trabajo en aulas del secundario, en la formación continua y capacitación a docentes, jornadas institucionales, congresos y jornadas dedicadas a la enseñanza de la Filosofía, foros nacionales e internacionales y, fundamentalmente, observaciones de clases.

Pero el pesimismo de la razón, como nos enseño aquel maestro italiano, no es condición suficiente para abandonar el optimismo de la voluntad. Que no tengamos hoy un cuerpo profesoral con las condiciones de formación filosófica, pedagógica y política que sea capaz de provocar aprendizajes filosóficos significativos no invalida nuestro esfuerzo por instalar un gran debate en torno a este problema, fundamentalmente porque nada indica que un adolescente no sea capaz de interrogarse e interrogar al mundo en que vive con una actitud filosófica. Somos los/as profesores/as quienes debemos recuperar esa actitud que nos movió a estudiar Filosofía. Especialmente contra un discurso académico que desalienta casi sistemáticamente, podríamos decir, dos cosas: que en la propia universidad o en el profesorado se puede producir Filosofía mientras se la estudia y que la enseñanza de la Filosofía en la escuela secundaria es algo menos que un destino de segunda calidad profesional.

\section{El problema de la autonomía del profesor}

¿Hacia dónde encaminar entonces la tarea de renovación de la enseñanza de la Filosofía?, ¿en base a qué principios filosóficos y pedagógicos debemos construir la propuesta formativa para los futuros profesores de Filosofía?, ¿cómo hacemos para que la enseñanza de la Filosofía se vuelva cada vez más filosófica y menos "técnica"? Si hacer Filosofía en un aula es el nombre posible para una actividad del pensamiento que posibilite a cualquier sujeto pensar sus propios posicionamientos subjetivos, ¿qué haría posible que alguien piense, advierta, cómo se posiciona subjetivamente dentro de la institución educativa?, ¿de qué depende que esto ocurra?, ¿es necesaria una intervención exterior al sujeto para que esto ocurra?, ¿cuál sería la legitimidad de esta intervención externa?, ¿cuál es el espacio para lo no sabido en una clase de Filosofía?, ¿se puede pensar en algún tipo de "encuadre" para enseñar Filosofía, como por ejemplo asumir una posición de no saber como punto de partida?, un posicionamiento subjetivo se- 
mejante, ¿es una cuestión metodológica o conceptual?, ¿habría algún dispositivo confiable, en el sentido de que no reemplace o interprete al otro?, ¿dónde se gesta lo que debe enseñarse en la escuela?, y finalmente ¿la institución escuela admitiría unas prácticas semejantes?

Tomando en consideración lo que hemos dicho sobre creciente autonomía y consolidación de un campo propiamente filosófico alrededor de la cuestión de la enseñanza de la Filosofía, consideramos que estamos en condiciones, actualmente, de tomar una distancia crítica de la habitual demanda de "métodos" o "dispositivos" de enseñanza que reclaman con frecuencia los aspirantes al profesorado. Si bien la transición de la condición de estudiante a profesor de Filosofía genera situaciones de incertidumbre y ansiedad, no es menos cierto que el conjunto de problemas prácticos que los futuros profesores y profesoras deben afrontar no se resuelven simplemente con el hallazgo de la "clave didáctica" que garantice una correcta enseñanza. Lo que más le suele costar a un estudiante próximo a convertirse oficialmente en profesor no es tanto el manejo de los textos, el vínculo con los alumnos o la dosificación de las actividades, como si lo es establecer una relación propia con la Filosofía. Y el recurso a la prescripción metodológica es una tentación pedagógica harto frecuente que quisiéramos eludir. Los profesores/as solemos estar ávidos de esta información sobre nuevos "recursos didácticos", especialmente en el tiempo que nos toca vivir, donde la escuela encuentra una fuerte y desleal competencia en los medios masivos de comunicación y en la informática del entretenimiento. Como suele decir Alejandro Cerletti, el imperativo categórico pedagógico que pesa sobre los maestros contemporáneos es el no aburrirás.

La idea que proponemos, entonces, es que el espacio de la didáctica especial de la Filosofía tanto como el de las prácticas docentes, son ocasiones pertinentes para que los estudiantes reflexionen sobre sí mismos y sobre su vínculo con la Filosofía. La reconstrucción de los motivos por los cuales se quiere enseñar Filosofía, tanto como lo que se supone que se hará en nombre de ella, promueven en los futuros profesores, una tarea de elucidación, es decir, de pensar lo que se hace y saber lo que se piensa. Tal como lo señalase el propio G. Obiols en el artículo que hemos tomando como referencia, el estudiante próximo a graduarse como profesor de Filosofía debe examinar "sus sentimientos" respecto de la profesión docente. Al menos para tener en claro si la misma constituirá algo más que, como confesara E. Gilson, "una molestia" o "un mal menor".

Como aporte final a esta reflexión sobre la tarea de formar profesores de Filosofía (aunque extensible a la formación de cualquier profesor) creemos que serían defendibles dos principios muy sencillos que tomo prestados de $\mathrm{C}$. Castoriadis: 
- Todo proceso educativo que no apunte a desarrollar al máximo la actividad propia de los alumnos es malo;

- Todo sistema educativo incapaz de proveer una respuesta razonable a la pregunta de los alumnos: ¿y por qué tenemos que aprender esto?, es defectuoso. (CASTORIADIS, 2008, p. 120)

Podría incluso fundamentarse un ensayo de propuesta filosófico-pedagógica para el espacio de la didáctica especial y la residencia docente, desde los conceptos de autonomía y elucidación sobre los que largamente trabajó Castoriadis. Las múltiples determinaciones que constituyen nuestra subjetividad son enfocadas por él en términos de construcción de significaciones imaginarias. La autonomía es alcanzada por nosotros cuando devenimos en sujetos reflexivos, es decir, cuando podemos cuestionar las significaciones imaginarias sociales que incorporamos desde que llegamos al mundo. El establecimiento de un discurso propio, es decir, un discurso no alienado, es lo que él denomina como proyecto de autonomía, entendiendo que la elección de un proyecto identificatorio y una interpretación de nuestra propia historia, es lo que da sentido a nuestro pasado y a nuestro porvenir. Romper la clausura a nivel del pensamiento es lo que Castoriadis entiende como la tarea de la Filosofía. En esa tarea, que él llama elucidación, de lo que se trata es del intento de pensar lo que se hace y de saber lo que se piensa.

Es evidente que no basta con construir un saber disciplinar; tampoco alcanza con construir un saber pedagógico para encarar una enseñanza de la Filosofía de modo significativo para los alumnos y para nosotros. Es necesario construir también otro saber de igual importancia: un saber sobre nosotros mismos. Por supuesto que saber sobre la historia de la Filosofía es una condición necesaria para enseñarla. Pero no suficiente. A diferencia de otros modos de comprender la relación entre individuo y sociedad, Castoriadis entiende que si bien nuestra subjetividad es producto de lo histórico-social, las determinaciones que ello implica no son condicionantes absolutos. De hecho, el proyecto de autonomía del sujeto, más allá de elementos preexistentes, implica el surgimiento de una novedad radical. La autonomía no es la eliminación total del discurso del Otro, no sabido como tal. Es la instauración de otra relación entre el discurso del Otro y el discurso del sujeto. En esta dirección, poder preguntarnos como profesores/ as de Filosofía a quién se enseña, con qué propósito, qué relaciones establecer entre objetivos, contexto y contenidos, apunta a poner en cuestión esas significaciones imaginarias sociales. Hay una capacidad instituyente colectiva en el trabajo sobre estas preguntas en el profesorado. Las instituciones por las que transcurre nuestra vida son justamente una red simbólica socialmente sancionada en la que se combinan componentes funcionales e imaginarios. Cuando el sujeto 
es heterónomo, las legalidades institucionales se le presentan como un todo instituido inmodificable, quedando ocultas las fuentes del poder, volviéndose de hecho extraño a los sujetos.

La formación de profesores en las universidades y en los profesorados suele garantizar, en el mejor de los casos, el saber disciplinar y el saber pedagógico. El desafío más inquietante es acompañar la construcción del otro saber: el de sí mismos. Provocar la autonomía del otro, sabemos, es un imposible. Pero sin embargo si es posible que el aula se convierta en un espacio para la reconstrucción de las subjetividades. Un espacio para ensayar decisiones sobre nuestra relación con el mundo y con nuestros semejantes. Si al menos en esto no creyéramos, bastaría con poner llave a las escuelas y marchar a la búsqueda de otras experiencias.

\section{REFERÊNCIAS}

BERTTOLINI, M. La educación filosófica para la reconstrucción de las subjetividades. La discusión filosófica como dispositivo propio y apropiado. En: KOHAN, W. et al. Teoría y práctica en filosofía con niños y jóvenes: experimentar el pensar, pensar la experiencia. Buenos Aires: Novedades Educativas, 2006, p. 267-278.

CASTORIADIS, C. La institución imaginaria de la sociedad. Buenos Aires: Tusquets, 2007. mar, 2008.

. Psicoanálisis y política. En: . El mundo fragmentado. La Plata: Terra-

CERLETTI, A. La enseñanza de la filosofia como problema filosófico. Buenos Aires: Libro del Zorzal, 2008. 2010.

; RUGGIERO, G. (Eds.). Enseñar filosofía en los límites. Los Polvorines: UNGS,

GALLO, S. et al. (Org.). Filosofía do ensino de Filosofía. Petrópolis: Vozes, 2003.

LANGÓN, M. Filosofía do ensino de filosofía. En: GALLO, S. Filosofía do ensino de Filosofía. Petrópolis: Vozes, 2003.

OBIOLS, G. Una misión casi imposible: enseñar a enseñar filosofía. En: OBIOLS, G.; FRASSINETI, M. La enseñanza filosófica en la escuela secundaria. Buenos Aires: A-Z, 1991. 
; AGRATTI, L. Concepciones de la Filosofía y enseñanza de la Filosofía en la escuela secundaria: un análisis a través de los programas y los libros de texto. En: ; FRASSINETI, M. La enseñanza filosófica en la escuela secundaria. Buenos Aires: A-Z, 1991.

; RABOSSI, E. La Filosofia y el filosofar. Buenos Aires: Centro Editor de América Latina, 1993. Educativas, 2000.

. La enseñanza de la Filosofía en debate. Buenos Aires: Novedades

VICARI, P. Enseñanza de la Filosofía y curriculum provincial. Análisis crítico de las reformas curriculares propuestas por la Dirección General de Escuelas (Prov. Bs. As.) para la enseñanza de la filosofía en el nivel Polimodal. En: CERLETTI, A. (Comp.). La enseñanza de la Filosofía en perspectiva. Buenos Aires: Eudeba, 2009

Texto recebido em 13 de fevereiro de 2012.

Texto aprovado em 30 de junho de 2012. 